Ann. Biol. anim. Bioch. Biophys., I976, 16 (6), 807-819.

\title{
ÉTUDE DE QUELQUES ENZYMES DU PLASMA SÉMINAL DE COQ
}

\author{
Madeleine SERVOUSE, M. J. PETITJEAN* et A. J. ROSENBERG \\ Laboratoire de Biochimie 1. U.E.R. Sciences, \\ 40, avenue du Recteur Pineau, \\ 86022 Poitiers \\ *I. N.R. A., Station Expérimentale d'Aviculture du Magneraud \\ 17700 Surgères
}

\section{RÉSUMÉ}

Le plasma séminal de coq possède des enzymes à la fois solubles et particulaires. Les enzymes particulaires accompagnent les spermatozoïdes au cours du fractionnement du sperme par centrifugation, et leur étude doit permettre de préciser leur rôle dans la fécondation.

Les enzymes particulaires peuvent être séparées des enzymes solubles ( $\beta$-N-Acétyl-glucosaminidase, Nucléotide pyrophosphatase, Lysozyme) par ultracentrifugation ( 22 ooo $g$ - I heure) ou filtration sur gel SEPHADEX G 200 ; dans ce dernier cas, le diagramme d'élution des substances absorbant à $280 \mathrm{~nm}$ présente trois pics, contenant successivement les protéines particulaires, les protéines solubles et l'acide urique. L'examen en microscopie électronique du culot - I22 000 $g$ met en évidence des vésicules arrondies ( $d=0,1$ micron). Ces vésicules possèdent une ATPase $\mathrm{Métal}^{++}$dépendante et une phosphatase acide. Les ions $\mathrm{Ca}^{++}$ont un effet activateur supérieur à celui des ions $\mathrm{Mg}^{++}$sur l'hydrolyse de l'ATP à $\mathrm{pH} 8$.

Cette fraction ne semble pas d'origine sanguine. Elle ne provient pas d'une altération subie par les flagelles des spermatozoïdes, puisque sa quantité diminue dans le plasma lorsqu'on augmente les risques de lésion par centrifugation. Elle serait une sécrétion de l'appareil reproducteur.

\section{INTRODUC'TION}

Le plasma séminal de coq est un milieu isotonique qui joue un rôle protecteur à l'égard des spermatozoïdes qui doivent y survivre plusieurs jours in vivo. C'est un produit de sécrétions d'origines diverses : testicules, cellules bordant les différents conduits du système reproducteur. Il s'y ajoute souvent, au cours de la récolte artificielle du sperme, une sécrétion accessoire, le liquide transparent, considéré comme un dialysat de plasma sanguin. Il contient également des produits du métabolisme des spermatozoïdes et parfois des spermatozoïdes en dégénérescence. L'étude de ses 
composants est rendue difficile par le fait même que le plasma est obtenu par la filtration ou la centrifugation. La centrifugation peut endommager le spermatozoïde et entraîner selon MANN (I964), la libération d'une partie de ses protéines dans le plasma. Cependant, l'étude du plasma peut apporter des informations sur les facteurs nécessaires à la conservation des spermatozoïdes et éventuellement au maintien de leur pouvoir fécondant.

Le plasma séminal peut en particulier hydrolyser aussi bien la liaison pyrophosphate des Nicotinamide-Adénine-Dinucléotides, oxydé et réłuit, que celle en position $\beta$ de 1'A'TP. Nous avons essayé de séparer ces deux activités enzymatiques et de préciser la provenance de l'une d'elles, l'ATPase.

\section{MATÉRIEI E'T MÉTHODES}

\section{1. - Prélèvements}

Les coqs appartiennent à une souche de type " ponte " issue en grande partie d'animaux de la race $R$ hode Island rouge. Ils sont entretenus en batteries individuelles dans un local conditionné ( 6 heures de lumière/8 heures d'obscurité par jour ; température régulée aux environs de $+\mathrm{I} 5^{\circ} \mathrm{C}$ ).

Le sperme est prélevé suivant la méthode de Burrows et QuinN (I937) à la température ambiante. Il est immédiatement centrifugé à basse vitesse durant I $O^{\prime}$. Le plasma séminal décanté est conservé congelé $\left(-18^{\circ} \mathrm{C}\right)$; il constitue la fraction " plasma brut ". Avant la congélation, certains échantillons ont été filtrés sur Millipore de diamètre $0,45 \mu$, de façon à éliminer tout débri de spermatozoïdes; ils sont qualifiés de "plasma brut filtré ".

Les sérums sont obtenus à partir d'échantillons de sang prélevés dans la veine alaire.

\section{2. - Activités enzymatiques}

a) ATPase (E.C : 3.6.r.3.).

Les conditions expérimentales seront précisées dans les tableaux de résultats. La rech de $1^{\prime}$ ATPase s'effectue à $25-30^{\circ} \mathrm{C}$, à un $\mathrm{pH}$ compris entre 8 et 8,5 dans un tampon Tris- $\mathrm{HCl}$ o, $\mathrm{I}$ M. L'ATP-Na $a_{2}$ est dissous dans ce même tampon; les ions $\mathrm{Ca}^{++}$et $\mathrm{Mg}^{++}$sont introduits sous forme de chlorure.

La réaction est initiée avec l'ATPase et bloquée avec l'acide trichloracétique 6,66 p. Ioo; après une centrifugation et neutralisation du surnageant avec $\mathrm{NaOH}$, l'ADP est recherché selon la méthode d'Adam (r963).

b) Nucléotide pyrophosphatase (E : C.3.6.r.9.).

Le substrat est $\mathrm{NADH}$, à une concentration comprise entre 2 et $3,0 \cdot 10^{-3} \mathrm{M}$. Cette concen. tration sera précisée à chaque expérience. La recherche de l'activité s'effectue à $25^{\circ} \mathrm{C}$ dans du tampon Tris-HCl o, I M; $\mathbf{p H ~ 8 , 5 ~ s u i v a n t ~ l a ~ t e c h n i q u e ~ d e ~ M A N C E A U ~ ( 1 9 7 2 ) . ~}$

\section{c) Activité phosphomonoestérasique (E.C : 3.I.3.)}

Différents substrats ont été utilisés :

- Le glucose-6-phosphate $5,0 \cdot \mathrm{IO}^{-2} \mathrm{M}$ dans un tampon citrate $0, \mathrm{I} \mathrm{M}, \mathrm{pH} 6,5$ à $30^{\circ} \mathrm{C}$ : recherche de la glucose-6-phosphatase (E.C : 3.1.3.9.). HARPER (I963).

- l'AMP 2,5 I $^{-2} \mathrm{M}$, en présence de $\mathrm{Mg}^{++} 8,0 \cdot \mathrm{IO}^{-2} \mathrm{M}$, dans un tampon $\mathrm{Tris}-\mathrm{HCl}$ o, $\mathrm{M}$, pH 8,5 ou citrate o, I M, pH 6,5 à $3^{\circ} \mathrm{C}:$ - recherche de la $5^{\prime}$ nucléotidase (E.C : 3.I.3.5.) HePPEL and HiLmoe (1955).

- Le paranitrophénylphosphate de sodium : $\mathbf{1}, 0 \cdot 10^{-2} \mathrm{M}$, dans un tampon citrate $\mathrm{o}, \mathrm{I} \mathrm{M}$,

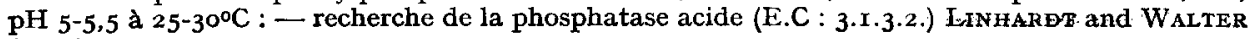
$(1963)$. 
d) Activité $\beta$-N-acétyl glucosaminidasique (E.C : 3.2.1.30.).

Le substrat est le paranitrophényl $\mathrm{N}$-acétyl- $\beta$-D-glucosaminide $2,4 \cdot 10^{-3} \mathrm{M}$ dans un tampon citrate o, $\mathrm{M}, \mathrm{pH}$ 3,8. TARENTINo and MALgY (1972).

e) Lysozyme (E.C : 3.3.r.1.).

Le substrat est une suspension de Micrococcus Lysodeitikus à o, I $5 \mathrm{mg}$ par $\mathrm{ml}$ de tampon phosphate $0, \mathrm{I} \mathrm{M}, \mathrm{pH} 7$. La diminution de turbidité est évaluée à $45^{\circ} \mathrm{nm}$.

\section{f) Expression des résultats.}

Pour chaque activité, à l'exception du lysozyme, l'unité d'enzyme correspond à la quantité d'enzyme qui libère une micromole du produit dosé, par minute, dans les conditions de l'expérience.

L'unité d'activité du lysozyme est définie comme étant la quantité d'enzyme qui provoque une variation de la densité optique de o,oor par minute dans les conditions expérimentales cidessus ( $\mathrm{pH} 7$ et $25^{\circ} \mathrm{C}$ ).

$$
\text { 3. - Protéines }
$$

Elles sont dosées :

- soit sur le culot de précipitation trichloracétique par la méthode du microbiuret (GoA I953), (I94I).

- soit sur l'éluat lors de la filtration sur gel par la méthode de WARBURg and CHRISTIAN

$$
\text { 4. - Acide urique }
$$

Il est dosé spectrophotométriquement par la méthode à l'uricase (PRAEToRIUS I963).

\section{5. - Chromatographie sur colonne de gel Sephadex}

Le gel est préparé dans le tampon d'élution Tris- $\mathrm{HCl} 0,02 \mathrm{M}, \mathrm{pH} 8$. L'échantillon est introduit sous un volume de $5 \mathrm{ml}$. La filtration est effectuée sur une colonne de $2,5 \times 30 \mathrm{~cm}$, à un débit de Io $\mathrm{ml} /$ heure et à $0-4^{\circ} \mathrm{C}$.

\section{6. - Microscopie électronique}

Le fixateur initial est une solution de glutaraldéhyde $2,5 \mathrm{p}$. Ioo dans un tampon phosphate $0, \mathrm{I} \mathrm{M}, \mathrm{pH} 7,4$. La fixation (I heure à $0^{\circ} \mathrm{C}$ ) est suivie de deux lavages avec du saccharose $0,23 \mathrm{M}$ en solution dans le tampon précédent. Le tissu est traité à l'acide osmique I p. Ioo pendant I heure, déshydraté dans l'acétone et inclus dans l'araldite.

\section{RÉSULTATS ET DISCUSSION}

\section{I. - Isolement de l'ATPase}

La première technique utilisée fut l'ultracentrifugation.

Le plasma séminal " brut " est opalescent; au moment de la décongélation, on observe la présence d'un culot dans le fond du tube. Si ce plasma brut subit une centrifugation sous 122 ooo $g$ pendant trente minutes, l'opalescence disparaît presque totalement ; cette même force centrifuge, relative, appliquée soixante minutes, rend le plasma absolument limpide et la fraction sédimentée offre un aspect visqueux.

L'activité ATPasique a été recherchée parallèlement dans le plasma " brut " et les deux fractions isolées par centrifugation. Après une heure de centrifugation 


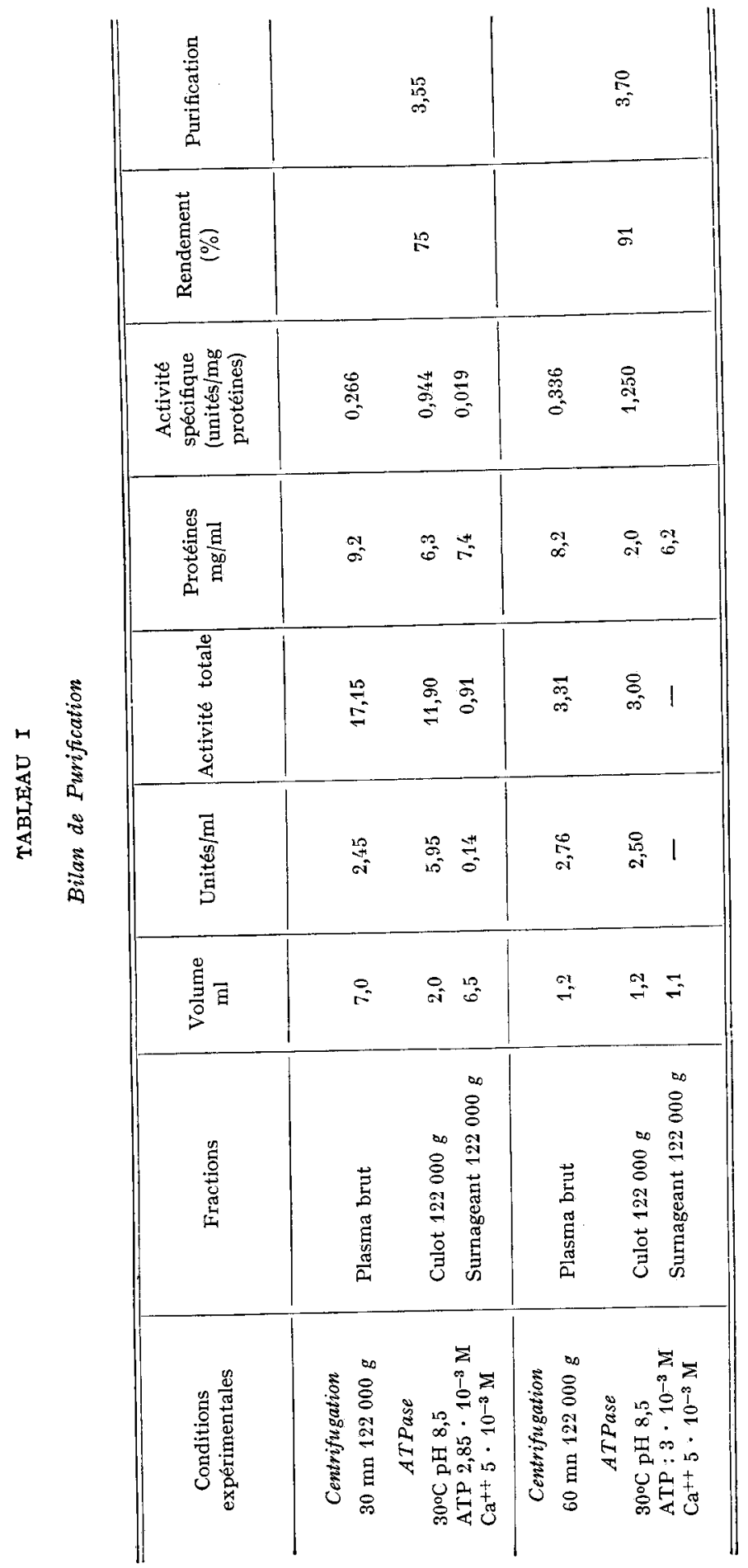




\section{TABLEAU 2}

Localisation de la dinucléotidepyrophosphatase et de l'ATPase dans les trois fractions des diagrammes d'élution 1 et 1 bis

Activités des fractions du diagramme : le pic d'élution I a subi une centrifugation à $122000 \mathrm{~g}$.

- NADH $2,5 \cdot \mathrm{IO}^{-3} \mathrm{M}$,

$-\mathrm{ATP}_{3} \cdot \mathrm{I0}^{-3} \mathrm{M}-\mathrm{Mg}^{++} 5 \cdot \mathrm{IO}^{-3} \mathrm{M}-\mathrm{pH} 8,5-30^{\circ} \mathrm{C}$.

\begin{tabular}{|c|c|c|c|c|c|c|c|}
\hline & \multicolumn{3}{|c|}{ Protéines } & \multicolumn{2}{|c|}{$\begin{array}{c}\text { NADH- } \\
\text { Pyrop hosphatase }\end{array}$} & \multicolumn{2}{|c|}{ ATPase } \\
\hline & D.O. 280 & $\mathrm{mg} / \mathrm{ml}$ & $\underset{\text { fraction }}{\mathrm{mg} /}$ & $\begin{array}{l}\text { unités/ } \\
\text { fraction }\end{array}$ & $\begin{array}{l}\text { activité } \\
\text { spécifique }\end{array}$ & $\begin{array}{l}\text { unités/ } \\
\text { fraction }\end{array}$ & $\begin{array}{l}\text { activité } \\
\text { spécifique }\end{array}$ \\
\hline Fraction $I$ & 0,700 & 0,241 & 7,475 & 0,0375 & - & 2,415 & 0,67 \\
\hline $\begin{array}{l}\text { Culot } 122000 \mathrm{~g} \ldots \\
\text { Surnageant } \ldots \ldots\end{array}$ & & $\begin{array}{l}0,116 \\
0,125\end{array}$ & $\begin{array}{l}3,600 \\
3,875\end{array}$ & $\begin{array}{c}\text { non } \\
\text { dosable }\end{array}$ & 一 & 2,170 & 0,56 \\
\hline $\begin{array}{l}\text { Fraction } I I \\
\text { Surnageant } \\
122000 \mathrm{~g} \ldots \ldots\end{array}$ & 0,330 & 0,575 & 23,000 & 2,8 & 0,122 & $\begin{array}{c}\text { non } \\
\text { dosable }\end{array}$ & - \\
\hline Fraction $I I I$ & $>1,0$ & \multicolumn{2}{|c|}{ non précipitables } & \multicolumn{2}{|c|}{ absente } & \multicolumn{2}{|c|}{ absente } \\
\hline
\end{tabular}

Activités des fractions du diagramme I bis.

- NADH 2,4 $\cdot \mathrm{IO}^{-3} \mathrm{M}$,

$-\operatorname{ATP}_{2,85} \cdot \mathrm{IO}^{-3} \mathrm{M}-\mathrm{Ca}^{++} 5 \cdot \mathrm{IO}^{-3} \mathrm{M}-\mathrm{pH} 8,5-30^{\circ} \mathrm{C}$.

\begin{tabular}{|c|c|c|c|c|c|c|c|}
\hline & \multicolumn{3}{|c|}{ Protéines } & \multicolumn{2}{|c|}{$\begin{array}{c}\text { NADH- } \\
\text { Pyrophosphatase }\end{array}$} & \multicolumn{2}{|c|}{ ATPase } \\
\hline & D. 0.280 & $\mathrm{mg} / \mathrm{ml}$ & $\underset{\text { fraction }}{\mathrm{mgl}}$ & $\begin{array}{l}\text { unités/ } \\
\text { fraction }\end{array}$ & $\begin{array}{l}\text { activité } \\
\text { spécifique }\end{array}$ & $\begin{array}{l}\text { unités/ } \\
\text { fraction }\end{array}$ & $\begin{array}{l}\text { activité } \\
\text { spécifique }\end{array}$ \\
\hline Fraction I & 0,165 & 0,081 & 1,82 & $\begin{array}{c}\text { non } \\
\text { dosable }\end{array}$ & - & 0,7 & 0,38 \\
\hline Fraction II & 0,380 & 0,550 & 30,25 & 4,62 & 0,153 & $\begin{array}{c}\text { non } \\
\text { dosable }\end{array}$ & - \\
\hline Fraction III & $>1,0$ & \multicolumn{2}{|c|}{ non précipitables } & \multicolumn{2}{|c|}{ absente } & \multicolumn{2}{|c|}{ absente } \\
\hline
\end{tabular}


l'activité a disparu du surnageant et se retrouve en grande quantité dans le culot correspondant, mis en suspension pour cette recherche dans le tampon Tris pH 8-8,5 avec un homogénéiseur de Potter-Ellvejehm.

Le bilan de purification fait 1'objet du tableau $\mathbf{r}$.

La filtration du plasma brut sur gel SEPHADEX G 200 permet d'éluer trois classes de substances absorbant à $280 \mathrm{~nm}$ (diagramme I).

Ce diagramme peut se présenter sous 2 formes; la courbe I correspond à un plasma brut opalescent et la courbe $I$ bis à un plasma soumis à une force centrifuge de 122 ooo $g$ pendant 30 minutes. L'absorption du pic $I$, importante en $I$, se réduit en $I$ bis à un simple épaulement, précédant le deuxième pic.

L'acide urique a été identifié dans le dernier pic de chaque diagramme, toujours dépourvu de substance précipitant en milieu perchlorique ou trichloracétique, mais dont 1'absorption très élevée est due à la présence de ce dérivé purique.

La répartition des protéines, de l'activité dinucléotide pyrophosphatasique NADH-dépendante et de 1'activité ATPasique fut étudiée dans chaque pic (tab1. 2).

Chaque fraction, telle qu'elle est délimitée sur le graphique fit 1'objet d'une centrifugation 30 minutes $I 22$ ooo $g$; seule la fraction I de la figure $I$ donna naissance à un culot visqueux doué d'activité ATPasique. Les autres éluats furent utilisés tels quels. La fraction III contient la quasi-totalité de l'acide urique du plasma. I a fraction II, la plus riche en protéines contient l'enzyme nucléotide pyrophosphatasique (94 p. Ioo de 1'activité retrouvée). Cette enzyme n'hydrolyse pas les liaisons pyrophosphate de l'ATP et se trouve ainsi séparée de l'ATPase de la fraction I.

Les enzymes du plasma séminal de coq se présentent donc soit sous une forme soluble, soit une forme particulaire. C'est ainsi que la phosphatase acide, mise en évidence par BELL, et LAKE (I962), accompagne 1'ATPase dans la fraction I22 ooo $g$. Par contre, une autre enzyme hydrolytique, la $\beta$-N-acétyl glucosaminidase (McINDOE et LAKE I972) est dans le surnageant I22 000 $g$ qui contient également, outre la dinucléotide pyrophosphatase, le lysozyme (fig. 2).

L'ultracentrifugation, appliquée au plasma séminal d'oiseaux, recueilli suivant les techniques habituelles, permet de répartir les enzymes présentes entre deux fractions : soluble et particulaire.

Au cours de la filtration du plasma sur gel SEPHADEX G 200, la fraction particulaire est éluée avec le volume mort de la colonne, car elle ne peut pénétrer dans le gel. Cet éluat d'opalescence très variable, responsable d'une forte absorption dans 1'ultraviolet, a déjà été remarqué par STrRATII, (I970). L'absorption ne peut être imputée à une composition en amino-acides à forte prédominance en tyrosine et tryptophane, ni à l'acide urique (à l'état de traces dans ce pic) mais à un complexe lipoprotéique, responsable de l'opalescence, quand il est abondant. KICHEV et DANOV (I972) notent la présence d'une fraction lipoprotéique au point de départ de l'électrophorèse. Nous avons vérifié que 1'A'TPase ne pénètre pas dans le gel de polyacrylamide, à l'aide de la réaction au nitrate de plomb. La nature lipoprotéique de cette fraction, confirmée par un travail ultérieur, rappelle celle du gel décrit par PAUTARD (I962) et obtenu à partir des flagelles de spermatozoỉdes de poissons. Ce gel, qui se contracte en présence d'ATP et $\mathrm{Mg}^{++}$, ne put être préparé à partir des têtes de spermatozoìdes.

Le spermatozoïde de coq présente la même disposition fibrillaire que les spermatozoïdes des autres espèces, mais, à la différence de ceux des mammifères, la structure 

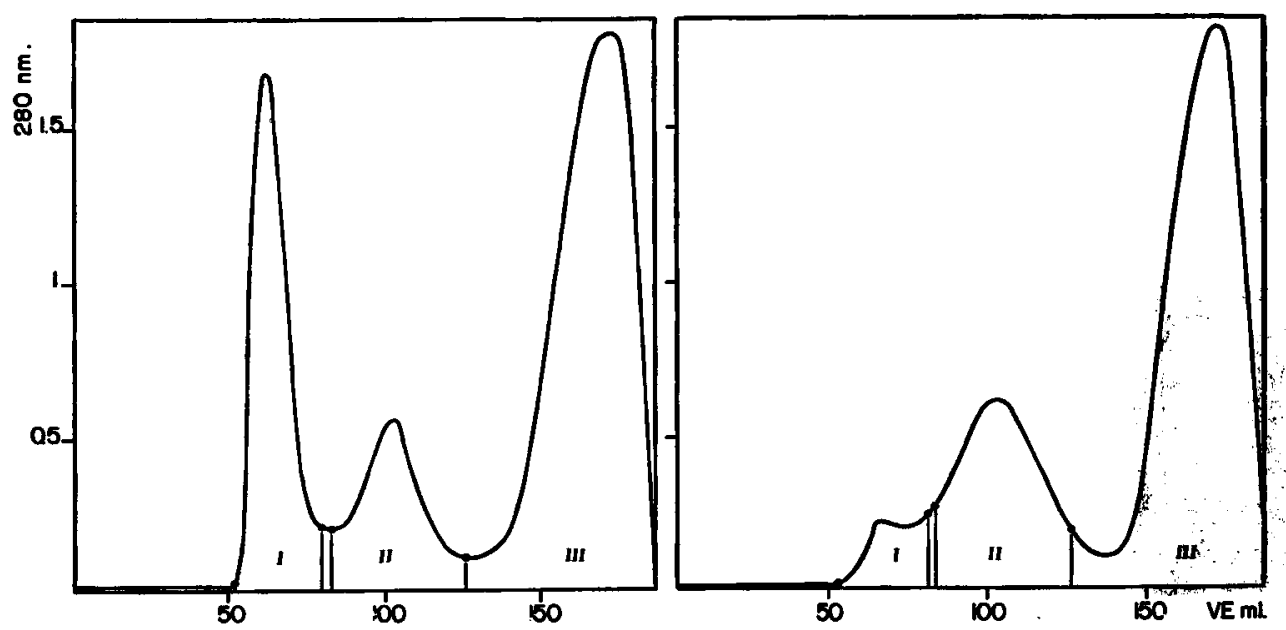

Frg. I. - Diagramme d'elution 1

Filtration du plasma séminal brut sur gel SEPHADEX G 200

FIG. I bis. - Diagramme d'elution $I$ bis

Filtration du plasma séminal, préalablement centrifugó

à $122000 \mathrm{~g}$, sur gel SEPHADEX G 200

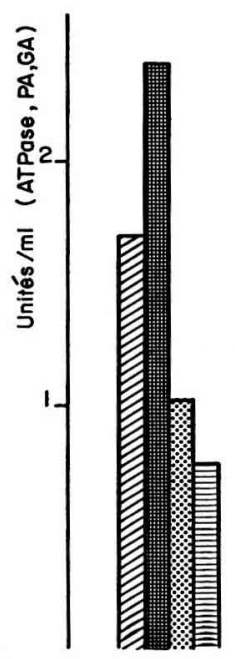

Plasma-brut
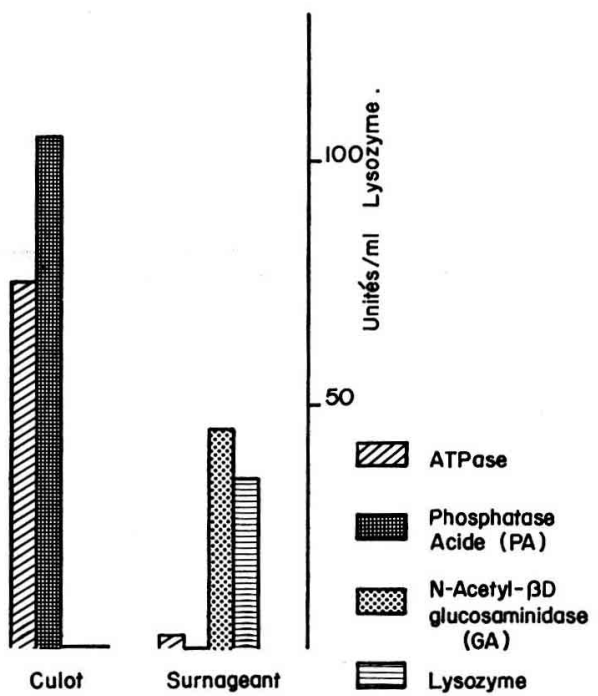

FIg. 2. - Localisation de l"ATPase, de la phosphatase acide, de la $N$-acétyl- $\beta-D$-Glucosaminidase et du Lysosyme

Les activités ont été recherchées à $25^{\circ} \mathrm{C}$,

- à $\mathrm{pH}: 8,45$ en présence de $\operatorname{ATP} 3 \cdot \mathrm{IO}^{-3} \mathrm{M}-\mathrm{Ca}^{++} 5 \cdot \mathrm{IO}^{-8} \mathrm{M}$

- à $\mathrm{pH}: 5,2$ en présence de p-nitrophénylphosphate $I \cdot I^{-2} \mathrm{M}$

- à $\mathrm{pH}: 3,75$ en présence de p-nitrophényl- $\mathrm{N}$-acétyl- $\beta$ - $\mathrm{D}$-giucosaminide $2,4 \cdot \mathrm{IO}^{-8} \mathrm{M}$

- à $\mathrm{pH}: 7$ en présence d'une suspension de Micrococcus Lysodeitikus à $0,12 \mathrm{mg} / \mathrm{ml}$. 
fibrillaire axiale est protégée par une enveloppe qui n'est pas de nature fibreuse; il s'agit d'une enveloppe amorphe, située sous la membrane cytoplasmique, qui va en s'effilant vers l'extrémité de la queue pour finalement disparaître. Elle peut être détruite par l'eau distillée aboutissant à la résolution du filament axial en fibrilles (MANN I964). Il faudrait alors admettre qu'au moment de l'éjaculation, 1'apport des sécrétions accessoires des tissus 1ymphoïdes détruit la gaine protectrice de certains spermatozoïdes par effet de dilution, ce qui entraîne une fragmentation des fibrilles porteurs de l'activité ATPasique (NEL,SON r962). L'ATPase libérée s'apparenterait alors à la myosine, protéine contractile responsable de l'activité ATPasique du muscle.

\section{II. - Activité ATPasique : choix de l'ion métallique}

Le substrat de la myosine est 1'ATP-Ca++. Toutefois les ions calcium peuvent être remplacés par des ions magnésium, quand l'enzyme contient des traces d'actine ou est contaminée par l'ATPase du réticulum sarcoplasmique ; $\mathrm{Mg}^{++}$devient alors un agent activateur en présence de traces de $\mathrm{Ca}^{++}$.

L'activité ATPasique du plasma séminal de coq a été recherchée en présence de ces deux ions, utilisés isolément ou mélangés : la concentration en ion retenue est celle qui correspond à une vitesse maximale d'hydrolyse de l'ATP par la myosine en présence de $\mathrm{Ca}^{++}$, à savoir une concentration égale ou légèrement supérieure à celle du nucléotide.

Le tableau 3 montre que $1^{\prime}$ ion $\mathrm{Ca}^{++} \mathrm{a}$ un effet activateur très net : 1'activité se trouve multipliée par I $_{5}$ quand la source d'enzyme est le culot de centrifugation. Si l'on s'adresse au plasma initial, le facteur de multiplication n'est plus égal qu'à 5 .

TABIEAU 3

Variation de l'activité ATPasique spécifique du plasma brut et du culot $122000 \mathrm{~g}$ en fonction de l'ion métallique à $\mathrm{pH}: 8 \mathrm{ATP}: 2,8 \cdot 10^{-3} \mathrm{M} 30^{\circ} \mathrm{C}$

\begin{tabular}{|c|c|}
\hline Addition d'ion métallique divalent & $\begin{array}{l}\text { Activité } \\
\text { atpasique spécifique }\end{array}$ \\
\hline 1. Plasma brut & \\
\hline 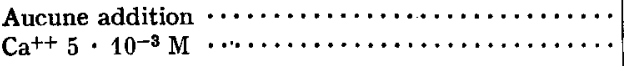 & $\begin{array}{l}0,05 \\
0,27\end{array}$ \\
\hline 2. Culot $122000 \mathrm{~g}$ & \\
\hline 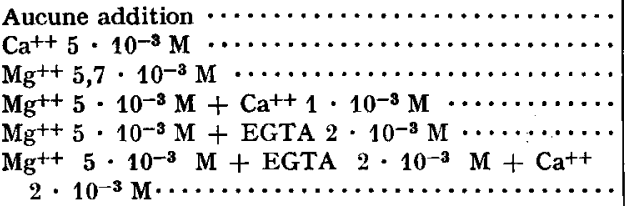 & $\begin{array}{l}0,065 \\
1,01 \\
0,75 \\
0,76 \\
0,80 \\
0,7^{\prime}\end{array}$ \\
\hline
\end{tabular}


Dans ce cas, même sans addition de $\mathrm{Ca}^{++}$, l'enzyme fonctionne grâce aux ions normalement présents dans le plasma. Si l'on compare les effets activants des deux ions, $\mathrm{Mg}^{++}$et $\mathrm{Ca}^{++}$à $\mathrm{pH} 8,1^{\prime}$ ion $\mathrm{Ca}^{++} \mathrm{a}$ un effet supérieur à celui de $\mathrm{Mg}^{++}$. Toutefois, nous avons voulu vérifier que l'effet activant du magnésium n'était pas dû aux traces de calcium, contaminant 1'actomyosine de fibrilles (WEBER et HERZ I963). L'addition d'EGTA ne modifie pas l'activité ATPasique $\mathbf{M g}^{++}$dépendante. De plus, en mettant à profit la valeur élevée de la constante de complexation EGTA-Ca ${ }^{++}\left(5 \cdot 10^{10}\right.$ à $\left.25^{\circ} \mathrm{C}\right)$, on peut, à partir de quantités équivalentes de ces deux composés, obtenir une concentration en calcium libre très faible (voisine de $2 . \mathrm{IO}^{-7}$ M.L. ${ }^{-1}$ ), mais suffisante pour stimuler l'ATPase- $\mathrm{Mg}^{++}$myofibrillaire. Cette concentration, en présence de $\mathrm{Mg}^{++}$, est sans effet sur 1'activité du culot I22 $000 \mathrm{~g}$. Cette ATPase est donc sensible, soit aux ions $\mathrm{Ca}^{++}$, soit aux ions $\mathrm{Mg}^{++}$seuls. Cette propriété la différencie del'actomyosine du muscle, mais ne permet pas d'éliminer une origine flagellaire.

\section{III. - Étude de la fraction $122000 \mathrm{~g}$ au microscope électronique}

Cette fraction se présente sous forme de vésicules arrondies de diamètre voisin de $0, \mathbf{I}$ micron, possédant une membrane bien individualisée, à 3 couches dont une couche médiane plus claire, probablement de nature lipidique. Leur contenu est plus ou moins opaque au flux d'électrons. L'aspect vésiculaire et homogène de la préparation, la comparaison du diamètre moyen des vésicules ( $0, \mathbf{I}$ micron) à celui du flagelle, variant de 0,2 à 0,5 micron suivant le niveau de section (LAKE, SMITh et Young I968), tendent à infirmer la nature flagellaire de cette fraction, dont 1'aspect rappellerait celui d'un réticulum endoplasmique.

Toutefois, l'enzyme marqueur du réticulum, la glucose-6-phosphomonoestérase, présente à $\mathrm{pH} 6,5$ n'est pas détruite par incubation à $\mathrm{pH} 5$ : il s'agit donc d'une phosphatase non spécifique. L'enzyme marqueur des membranes plasmiques, la 5 ' nucléotidase (E.C : 3.I.3.5.), est totalement absente du plasma.

\section{IV. - Hypothèse sur l'origine de la fraction $122000 \mathrm{~g}$}

Nous avons choisi comme enzyme marqueur de cette fraction, 1'ATPase-Ca ${ }^{++}$ dépendante.

Pour toute protéine du plasma séminal, il y a plusieurs provenances possibles : le sang, le liquide testiculaire, les produits des sécrétions accessoires, les spermatozoïdes lésés, en particulier par la centrifugation. Le plasma a été filtré sur Millipore, ce qui élimine les débris de flagelles.

La recherche directe de l'activité ATPasique dans le sérum sanguin permettra d'élucider l'une des origines. Les deux autres vont être précisées en s'appuyant sur l'hypothèse suivante :

- ou l'enzyme provient d'une altération structurale du flagelle, liée à la centrifugation, et dans ce cas, la quantité passant en suspension dans le plasma doit varier proportionnellement à l'intensité de la force centrifuge responsable de la lésion,

- ou au contraire aucune corrélation positive entre ATPase et force centrifuge ne peut être mise en évidence. On peut conclure que l'enzyme accompagne normalement les spermatozoïdes dans le sperme éjaculé : elle peut alors provenir des sécréticus de l'appareil reproducteur. 


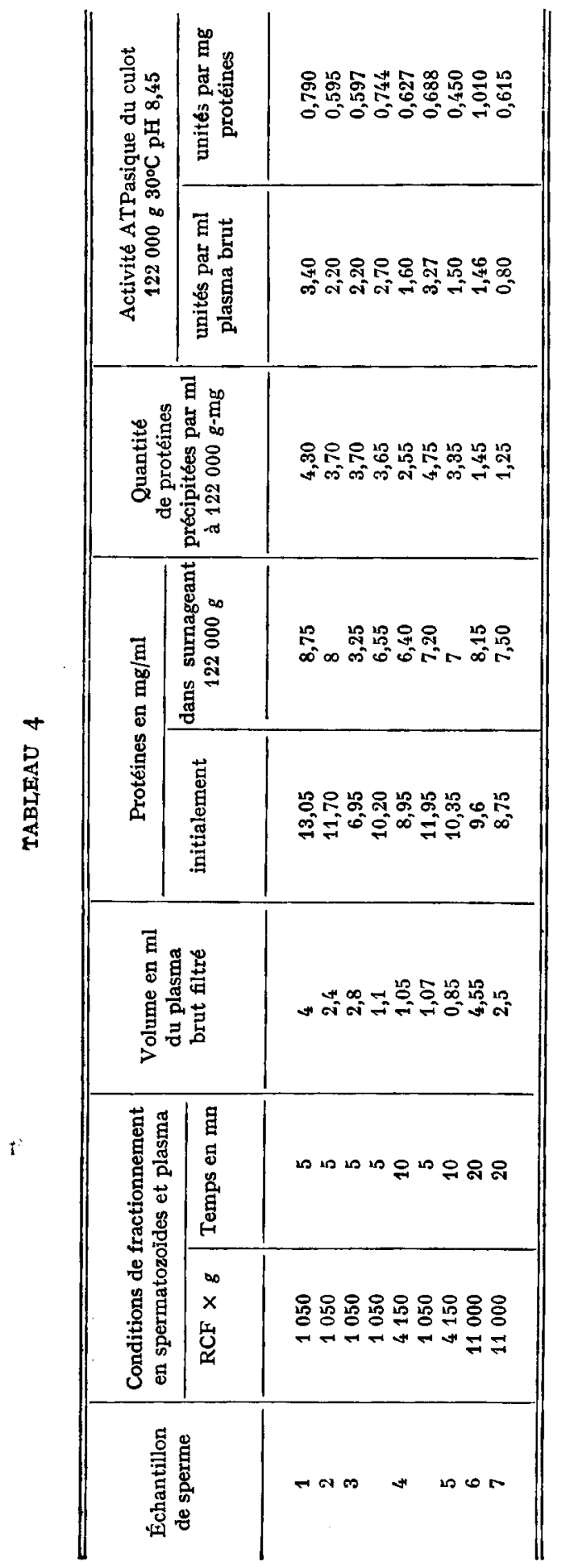


a) Recherche d'ATPase dans le sérum sanguin.

Le sérum sanguin est dépourvu d'activité ATPasique, malgré la présence d'un culot sédimentant à $122000 \mathrm{~g}$.

b) Recherche d'ATPase dans le plasma séminal.

On fait varier les conditions de fractionnement du sperme en ses composants, de façon à réaliser trois degrés de sédimentation, de force croissant dans l'ordre I $-\cdots---\rightarrow 3:$

$$
\begin{aligned}
& \mathrm{I}=5 \text { minutes }=\mathrm{I} 050 \mathrm{~g} \\
& 2=\text { I0 minutes }=4 \mathrm{I} 50 \mathrm{~g} \\
& 3=20 \text { minutes }=\mathrm{II} \text { o00 } \mathrm{g}
\end{aligned}
$$

L'étude du tableau IV montre que lorsque force centrifuge et temps augmentent de $x-\cdots---\rightarrow 3:$

I. Les quantités de protéines présentes dans I $\mathrm{ml}$ de plasma initial ont des valeurs de plus en plus faibles (à l'exception de l'échantillon 3 ).

2. La quantité de protéines sédimentées à $122000 \mathrm{~g}$ diminue.

3. L'activité ATPasique exprimée en micromoles d'ADP par minute et par millilitre de plasma initial diminue.

4. On observe par contre une valeur relativement constante de l'activité ATPasique lorsque l'on choisit comme référence pour son expression le poids des protéines correspondantes, sédimentées à $122000 \mathrm{~g}$; valeur moyenne : 0,68 ; écart-type : $0, \mathbf{I}_{5} 8$.

Le sang n'est pas à l'origine de la fraction protéique 122000 g. L'activité ATPasique diminue en outre quand la force centrifuge appliquée à l'échantillon de sperme augmente : ce qui est en désaccord avec l'hypothèse de la lésion structurale liée à la centrifugation.

Ces résultats peuvent être rapprochés de ceux de GARBERS et FirST (I97I) sur le plasma séminal de taureau et de verrat. Ce sont des éléments particulaires, élaborés par les glandes accessoires mâles, qui se déposeraient au cours de la centrifugation.

\section{CONCLUSION}

Le plasma séminal de coq, obtenu suivant les techniques habituelles, est une suspension de vésicules, de diamètre voisin de $0, I$ micron, responsables de son opalescence. La fraction particulaire est isolée par ultracentrifugation ou filtration sur gel SEPHADEX. Elle possède une double activité enzymatique : ATPase (E.C : 3.6.r.3.) $\mathrm{Ca}^{++}$et $\mathrm{Mg}^{++}$dépendante, phosphatase acide (E.C : 3.I.3.2.). Eille ne semble provenir ni du sang, ni d'une altération subie par les spermatozoïdes au cours du fractionnement, mais des sécrétions de l'appareil reproducteur.

Comme le coq est dépourvu d'organes accessoires mâles, ne peuvent contribuer à la composition du plasma que les sécrétions du testicule, de la région épididymaire et des canaux déférents. Or, l'étude de la structure épithéliale des canaux déférents 
corrobore nos résultats. LAKE (I962) y localise la phosphatase acide dans toutes les régions de l'épithélium, où elle serait le résultat d'une activité sécrétoire apocrine et holocrine des cellules. TINGARI et LAKE (I972) trouvent des lipides dans cette même région. TINGARI (1972) précise la structure des cellules sécrétrices non ciliées. Elles contiennent un abondant réticulum endoplasmique dont les vésicules sont gonflées de substance protéique, et des formations opaques, d'aspect vacuolaire, les lysosomes, où se trouverait la phosphatase acide. La matrice cytoplasmique renferme en outre des éléments fibrillaires et la présence de ces fibrilles, jointe à une riche innervation du tissu sous-épithélial, amène à envisager une fonction contractile de l'épithélium.

La fraction $122000 \mathrm{~g}$ pourrait être issue du réticulum endoplasmique de ces cellules, libéré par désintégration cellulaire, en même temps que des produits de sécrétions liquides. A l'appui de cette hypothèse, nous relevons un certain nombre de caractères communs à notre fraction et au réticulum endoplasmique du muscle et du cerveau, étudié par de MEIS (I970) et MARTONOSI (1972) : la localisation identique de 1'ATPase et de la phosphatase, l'absence de glucose-6-phosphatase, 1'aspect vésiculaire de la fraction, semblable à celui décrit par HASSEIBACH et EIfFIN (I967).

Or le rôle du réticulum sarcoplasmique a été précisé : les vésicules qui le constituent accumulent très rapidement les ions calcium en présence d'un excès d'ATP ; le calcium est libéré dans le sarcoplasme quand une excitation atteint la cellule musculaire et dès lors, il stimule l'activité ATPasique de la myosine. La contraction de 1'épithélium pourrait être le résultat d'un mécanisme analogue. La mise en évidence d'une " pompe à calcium " membranaire, au niveau du réticulum endoplasmique des cellules du canal déférent et au niveau de la fraction $122000 \mathrm{~g}$, serait en faveur de l'hypothèse de TINGARI (I972), qui suggère une contribution active de l'épithélium du tractus génital dans le mouvement des spermatozoïdes.

Cette fraction vésiculaire accompagne toujours les spermatozoïdes puisqu'elle sédimente avec eux. On peut alors se demander quelle est son influence sur le pouvoir fécondant des spermatozoïdes. Elle possède au moins deux enzymes, ATPase et phosphatase acide. Toutefois, leur fonction dans la fertilité est difficile à déterminer car elle doit être dissociée de celle remplie par les enzymes analogues des spermatozoïdes.

Reçu pour publication en avril 1976.

\section{SUMMARY}

A STUDY OF SOME SEMINAT, PLASMA ENZYMES OF THE COCKEREL

The seminal plasma of the cockerel contains enzymes which are either soluble or in particles. The latter migrate with the spermatozoa during centrifugation. This report attempts to understand their possible role in the fertilization process.

Particulate enzymes are separated from soluble enzymes ( $\beta$-N-Acetyl-glucosaminidase, Nucleotide pyrophosphatase, Lysozyme) by either gel filtration (Sephadex G-2oo) or ultracentrifugation (I $22000 \mathrm{~g}$, I hour).

In the first case, the elution diagram $(280 \mathrm{~nm})$ shows three peaks. The first mainly contains particulate enzymes, the second soluble proteins, and the third uric acid.

Electron microscopy examination of the 122 ooo $g$ residue shows round vesicles (diameter $=0.1$ micron) having a Metal ${ }^{++}$dependent ATPase and an acid phosphatase. Ca ${ }^{++}$ions have a stronger hastening effect than $\mathrm{Mg}^{++}$ions on ATP hydrolysis at $\mathrm{pH} 8$. 
This fraction does not seem to be of blood origin. It does not result from an injury of the spermatozoa flagelles since its quantity decreases in the plasma when risks of lesion are increased by centrifugation. It would therefore be a secretion of the reproductive system.

\section{RÉFÉRENCES BIBI,IOGRAPHIQUES}

AdaM H., I963. In Bergmeyer H. U., Methods of Enzymatic Analysis 573-577. Verlag Chemie-GMBHWinheim/Bergstr. Academic Press N. Y. and London.

Bell D. J., LAKe P. E., I962. Tissus components of the domestic fowl : 5 . Phosphomonoesterases in the seminal plasma of the cock. Biochem. J., 82, 277-28r.

Burrows W. H., Quins J. P., I937. The collection of spermatozoa from the domestic fowl and turkey. Poult. Sci., 16, I9-24.

De Meis L., Rubin Altschul $\quad$ B. M., Machado R. D., I97o. Comparative data of calcium transport in brain and skeletal muscle microsomes. J. Biol. Chem., 245, 1883-1889.

Garbers D. L., FIRST N. L., I97I. The importance of centrifugal forces in determining semen characteristics. J. Reprod. Fert., 25, 439-44I.

GoA J., I953. Scand. J. Clin., Lab. Invest. 5. 218. In Legget BaILeY J. Techniques in protein chemistry (1962) 294-295. Elsevier Publishing compagny. Amsterdam-London-New York.

HARPER A. E., I963. In Bergmeyer H. U., Methods Enzymatic Analysis 788-792. Verlag ChemieGMBH-Weinheim/Bergstr. Academic Press N.' Y. and London.

Hasselbach W., ElFiN L. G., I967. Structural and chemical asymmetry of the calcium transporting membranes of the sarcotubular system as revealed by electron microscopy. J. Uitrastruct. Res., 17, 598-622.

HePPel L. A., Hilmoe R. J., I955. 5-Nucleotidases in Colowick S. P. and Kaplan N. O. Methods in Enzymology., $\mathrm{Vol} 2,546-550$.

McIndoe W. N., LAKe P. E., r972. Hydrolytic enzymes of fowl semen. VII' Congr. intern. Reprod. Anim. Insemin. artif., Munich, 3, 2272-2274.

KIChev G., Danov D., r972. Microelectrophoretic study on cellogel of proteins glycol and lipoproteins in the seminal plasma and blood of the cock. VII Congr. intern. Reprod. Anim. Insemin. artif., Munich, 3 , 2275-2279.

LAKE P. E., I962. Histochemical demonstration of phosphomonoesterase secretion in the genital tract of the domestic fowl. J. Reprod. Fert., 3, 356-362.

LAKE P. E., Smirh W., Young D., x968. The ultrastructural of the ejaculated fowl spermatozoon. Q. Jl. Exp. Physiol., 53, 356-366.

Lindhart K., Walter K., I963. In Bergmeyer H. U., Methods of Enzymatic Analysis $783-785$. Verlag Chemie-GMBH-Weinheim/Bergstr. Academic Press N. Y. and London.

ManceaU D., r972. Communication personnelle.

MANN T., I964. The biochemistry of semen and of the Male Reproductive Tract. John Wiley. N. Y.

Martonosi A., I962. Biochemical and Clinical aspects of sarcoplasmic Reticulum function. In BRONNER F. and KLEINZELler A. Currents Topics in membranes and transport., vol 3, 83-197. Academic Press. N. Y. and London.

NelsoN L., I962. Cytochemical aspects of spermatozoan Motility in Bishop D. W. Spermatozoan Motility. I7I-I87. Amer, assoc. for Adv. Sci. Washington.

Pautard G. E., 1962. Biomolecular aspects of Spermatozoan Motility in Bishop D. W. Spermatozoan Motility. I89-232. Amer. Assoc. for Adv. Sci. Washington.

Praetorivs E. In Bergmeyer H. U., Methods of Enzymatic Analysis 500-501. Verlag Chemie-GMBHWeinheim/Bergstr. Academic Press. N. Y. and London.

Stratil A., I970. Studies on proteins of seminal fluid form the vasa differentia of the cock, Gallus Gallus L. Int. J. Biochem., 1, 728-734.

Tarentino A. L., Maley F., I972. N-Acetylglucosaminidase from Hen Oviduct. In Colowick S. P. ; Kaplan N. O, and Ginsburg F. Methods in Enzymology vol 28, 772-776. Academic Press N. Y. and London.

Tingari M. D., I972. The fine structure of the epithelial lining of the excurrent duct system of the testis of the domestic fowl. Q. Jl. Exp. Physiol., 57, 271-295.

TINGARI M. D., LAKE P. E., r972. Histochemical localization of glycogen mucopolysaccharides, lipids come oxydative enzymes and cholinesterases in the reproductive tract of the male fowl. J.Anat., 112 273-287.

Warburg O., Christian W., r94I. Biochem. Z. 310, 384 . In Colowick S. P. and Kaplan N. O. In Methods in Enzymology I957, 3, 45I-454. Academic Press New York.

Weber A., Herz R, 1963. The binding of Calcium to Actomyosin systems in relation to their Biological Activity. J. Biol. Chem., 288, 599-605. 